\title{
CONSTRUÇÃO DE UM BUNDLE PARA A INSERÇÃO DE CATETERES INTRAVENOSOS PERIFÉRICOS EM CRIANÇAS HOSPITALIZADAS
}

\author{
Taynara Bispo Conceição ${ }^{1}$; Luciano Marques dos Santos ${ }^{2}$; Gabriela Santos Silva ${ }^{3}$ \\ 1. Voluntário PEVIC/CNPq, Graduada em Enfermagem, Universidade Estadual de Feira de Santana, e-mail: \\ taynara_tatay@hotmail.com \\ 2. Orientador, Departamento de saúde, Universidade Estadual de Feira de Santana, e-mail: \\ lucmarxenfo@yahoo.com.br \\ 3. Participante do projeto Segurança do paciente pediátrico e sua família, Departamento de Nome, Universidade \\ Estadual de Feira de Santana, e-mail: gabisilva93@ hotmail.com
}

PALAVRAS-CHAVE: Cateterismo periférico; Segurança do paciente; Controle de infecções

\section{INTRODUÇÃO}

Na terapia intravenosa, a Cateterização Venosa Periférica (CIP) destaca-se como um dos procedimentos mais realizado no cuidado a criança hospitalizada, devido à facilidade na realização da técnica, e que consiste no acesso à corrente sanguínea através de dispositivos adequados, associados a uma seleção criteriosa do local da punção e de uma eficiente técnica de penetração da veia (Modes et al., 2011).

Em crianças é comum a CIP causar complicações locais no ato da técnica empregada. Neste contexto, requer inovações tecnológicas na prática do enfermeiro, respaldada em análise científica que contribua para melhorar os resultados na assistência, mais especificamente, a assertividade durante a inserção do CIP e o seu maior tempo de permanência no sítio de inserção (Avelar; Peterlini; Pedreira, 2013), potencializando o conforto da criança e do seu familiar acompanhante e a segurança do paciente.

Desta forma, o objeto deste estudo consiste em verificar a influência de um bundle de cateterização venosa periférica em crianças hospitalizadas, tornando necessário utilizar estratégias que possam colaborar com a redução de complicações mecânicas e infecções da corrente sanguínea. O bundle por sua vez, se constitui por um grupo de boas práticas, as quais são estabelecidas cientificamente. Individualmente, resultam em melhoria do cuidado e quando implementadas juntas apresentam um resultado ainda melhor. Essas práticas são agrupadas, apresentadas para uma equipe de cuidado, a qual deve cumprir os critérios do bundle (IHI, 2012).

Diante disso questiona-se: Quais são os cuidados clínicos realizados pelos profissionais de enfermagem antes, durante e após da inserção de cateteres intravenosos periféricos em crianças hospitalizadas? Cujo os objetivos foram: Avaliar os cuidados clínicos realizados por profissionais de enfermagem antes, durante e após a inserção de cateteres intravenosos periféricos em crianças hospitalizadas no HEC em Feira de Santana na 
Bahia e Elaborar um bundle para a inserção de cateteres intravenosos periféricos em crianças hospitalizadas no Hospital Estadual da Criança (HEC) em Feira de SantanaBahia.

\section{MATERIAIS E MÉTODOS}

Trata-se de um estudo de quantitativo, do tipo desenvolvimento de tecnologias, descritivo e exploratório. Foi desenvolvida em três etapas. $\mathrm{Na}$ primeira etapa foi realizada observação sistemática dos profissionais da enfermagem da Clínica Médica alas A e B do HEC objetivando avaliar os cuidados clínicos dos mesmos sobre CIP em crianças hospitalizadas. Na segunda etapa foi realizada a coleta de informações acerca das intervenções para a inserção de CIP em crianças, sendo consultados os manuais do Centers for Disease Control and Prevention (CDC), Infusion Nurses Society (INS) americana e brasileira, Agência Nacional de Vigilância Sanitária (ANVISA).

$\mathrm{Na}$ terceira etapa as informações coletadas, foram analisadas conforme suas convergências e divergências, sendo transformadas num bundle dividido em: cuidados antes, durante e após a CIP relacionados a higienização das mãos, precaução mínima de barreira, seleção do sitio de inserção, preparo do sitio de inserção, estabilização do hub do cateter e cuidados com a cobertura da CIP.

A população deste estudo foi composta por observação dos profissionais de enfermagem da Clínica Médica alas A e B do HEC durante a CIP. Amostra do estudo foi do tipo por conveniência.

Os dados foram tabulados em planilhas eletrônicas e analisados através do programa Statistical Package for Social Sciences (SPSS), versão 22, por meio do método de análise estatística descritiva. A análise dos dados foi realizada mediante frequência simples dos dados coletados através do formulário. Os dados foram descritos em variáveis categóricas e apresentadas em forma de tabela, onde foram calculadas frequências absolutas e percentuais.

Este estudo respeitou a Resolução 466/2012 do Conselho Nacional de Saúde sendo devidamente aprovado pelo Comitê de Ética na Pesquisa (CEP) da Universidade Estadual de Feira de Santana através do parecer 841. 612.

\section{RESULTADOS}


Tabela 1: Cuidados clínicos antes da inserção de cateteres intravenosos vasculares em crianças hospitalizadas em uma clínica médica de um hospital pediátrico no interior da Bahia. Feira de Santana - abril a junho, 2017.

\begin{tabular}{lll}
\hline \hline Variáveis & $\mathbf{N}=62(\%)$ & \\
\hline & Sim (\%) & Não (\%) \\
Realiza higienização das mãos? & $21(33,9 \%)$ & $41(66,1 \%)$ \\
Utiliza equipamento de proteção universal? & $51(82,3 \%)$ & $11(17,7 \%)$ \\
Utiliza touca & $32(51,6 \%)$ & $30(48,4 \%)$ \\
Utiliza luvas & $44(71 \%)$ & $18(29 \%)$ \\
Utiliza mascara & $19(30,6 \%)$ & $43(69,4 \%)$ \\
Inspeciona as condições da pele? & $62(100 \%)$ & ------ \\
Avalia as condições da rede venosa? (Visibilidade, profundidade e & $60(96,8 \%)$ & $2(3,2 \%)$ \\
trajeto) & & \\
Prefere veias dos membros superiores em comparação aos & $57(91,9 \%)$ & $5(8,1 \%)$ \\
membros inferiores? & $43(69,4 \%)$ & $19(30,6 \%)$ \\
Prefere veias do antebraço em relação ao braço? & & \\
\hline \hline
\end{tabular}

Fonte: Coleta de dados.

Tabela 2: Cuidados clínicos durante a inserção de cateteres intravenosos vasculares em crianças hospitalizadas em uma clínica médica de um hospital pediátrico no interior da Bahia. Feira de Santana - abril a junho, 2017.

\begin{tabular}{lll}
\hline \hline Variáveis & N =62 (\%) & \\
\hline $\begin{array}{lll}\text { Realiza antissepsia do sitio de inserção do cateter com 3 bolas de } \\
\text { algodão ou gaze estéril? }\end{array}$ & Sim (\%) & Não (\%) \\
$\begin{array}{l}\text { Quais soluções antissépticas utiliza no local de cateterização? } \\
\text { Clorexidina }\end{array}$ & $13(21 \%)$ & $(96,8 \%)$ \\
$\begin{array}{l}\text { Álcool à 70\% } \\
\text { Aguarda de 30 segundos a 1 minuto para a secagem da solução } \\
\text { antisséptica e realiza a tentativa de cateterização? }\end{array}$ & $49(79 \%)$ & \\
$\begin{array}{l}\text { Toca no sitio de inserção após a realização da antissepsia se for } \\
\text { necessário reavaliação da rede venosa? }\end{array}$ & $33(22,6 \%)$ & 48 \\
\hline \hline Fonte: Coleta de dados. & & 29 \\
\hline
\end{tabular}

Tabela 3: Cuidados clínicos após a inserção de cateteres intravenosos vasculares em crianças hospitalizadas em uma clínica médica de um hospital pediátrico no interior da Bahia. Feira de Santana - abril a junho, 2017. 


\begin{tabular}{|c|c|c|}
\hline Variáveis & $\mathbf{N}=62(\%)$ & \\
\hline & $\operatorname{Sim}(\%)$ & Não $(\%)$ \\
\hline Estabiliza o $h u b$ do cateter após o sucesso da cateterização? & $57(91,9 \%)$ & $5(8,1 \%)$ \\
\hline \multicolumn{3}{|l|}{ Coberturas utilizadas no cateter } \\
\hline IV - FIX ${ }^{\circledR}$ & $1(1,6 \%)$ & \\
\hline Filme transparente estéril & $1(1,6 \%)$ & \\
\hline IV - FIX ${ }^{\circledR}+$ Micropore $^{\circledR}$ & $27(43,5 \%)$ & \\
\hline IV - FIX ${ }^{\circledR}+$ Esparadrapo & $8(12,9 \%)$ & \\
\hline Micropore $^{\circledR}+$ Gaze estéril & $1(1,6 \%)$ & \\
\hline IV - FIX ${ }^{\circledR}+$ Micropore $^{\circledR}+$ Esparadrapo & $24(38,7 \%)$ & \\
\hline
\end{tabular}

Fonte: Coleta de dados.

\section{CONSTRUÇÃO DO BUNDLE}

A partir da análise dos estudos e das recomendações dos manuais, elaborou-se o bundle ou "pacote de cuidados" para inserção de cateter intravenoso periférico em crianças (FIGURA 1). O seguinte bundle possui cuidados destinados à relacionados a higienização das mãos, precaução mínima de barreira, seleção do sitio de inserção, preparo do sitio de inserção, estabilização do hub do cateter e cuidados com a cobertura da CIP.

Figura 01 - Bundle para inserção de cateter intravenoso periférico em crianças, Bahia, 2017.

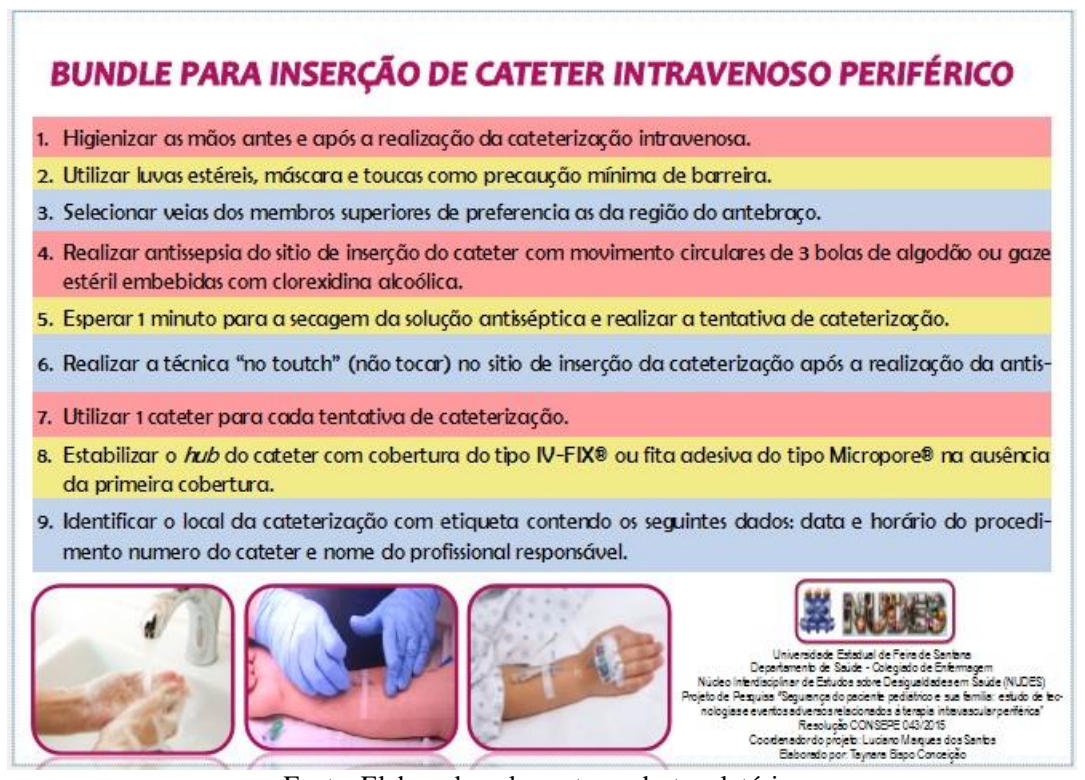

Fonte: Elaborado pelos autores deste relatório. 
Considerando os cuidados clínicos realizados antes da inserção de cateteres intravenosos vasculares em crianças hospitalizadas, o presente estudo apontou que em $66,1 \%$ das CIP, os profissionais não higienizaram as mãos antes da realização do procedimento. Estes dados são alarmantes e indicam que a não realização da lavagem das mãos por parte dos profissionais pode aumentar a possibilidade de contaminação do sítio de inserção do cateter, podendo assim evoluir posteriormente para infecção da corrente sanguínea (Martins et al., 2008). Esta intervenção na assistência à saúde é uma medida preventiva e está associada à redução das taxas de infecções e complicações relacionadas ao uso de cateteres intravenosos (Batista et el., 2014).

Outra prática importante de ser realizada antes da realização da CIP, é o cuidado que o profissional deve ter com a escolha do sitio de inserção do cateter. É recomendado que realize a inspeção das condições da pele, objetivando avaliar e escolher um local que não apresente lesões, devido a possibilidade de infecções e a avaliação das condições da rede venosa, quanto a visibilidade, trajeto e profundidade (INS, 2016; Oliveira; Bezerra; Pereira, 2008). Perante os fatos, os profissionais desse estudo seguem o que é recomendado pela literatura, pois em $100 \%$ das CIP observadas os profissionais inspesionaram as condições da pele do paciente e 96,8\%, avaliaram as condições da rede venosa, quanto visibilidade, profundidade e trajeto da veia selecionada para a CIP. A literatura recomenda que primeira inserção de cateter, deve sempre iniciar pela região mais distal, considerando as particularidades de cada fármaco e possibilidades de complicação, iniciando pelas veias da mão, antebraço e parte superior do braço abaixo a axila (Modes et al. 2011). Na atenção a criança hospitalizada, a literatura aponta a importância de definir o sítio de inserção do cateter periférico, mediante a ausência desse cuidado poderá comprometer a segurança da TIV e da terapia infusional, expondo a criança a eventos adversos e a novas CIP (Almeida et al., 2016).

O preparo do sitio de inserção é fundamental na prevenção de infecção (Lourenço; Ohara, 2010). No presente estudo, apenas 3,2\% das CIP observadas, os profissionais realizaram a técnica asséptica conforme é recomendado pela literatura. As soluções antissépticas devem ser a base de álcool, podendo ser o álcool $70 \%$ ou o gluconato de clorexidina 0,5 a $2 \%$ ou o PVPI alcoólico $10 \%$. Observamos que em apenas $21 \%$ das cateterizações, os profissionais utilizaram o gluconato de clorexidina 0,5 a $2 \%$ e $79 \%$ utilizaram o álcool a 70\% para a realização da antissepsia da pele.

Ainda sobre ao preparo do sitio de inserção, deve aguardar o tempo de ação do antisséptico e a completa secagem natural antes de realizar a CIP (INS Brasil, 2013). No 
presente estudo, apenas $22 \%$ das cateterizações observadas, os profissionais aguardaram a secagem da solução antisséptica antes de realizar a CIP.

É recomendado que aguarde de 30 segundos a 2 minutos para secagem e atingir a ação bactericida, antes da CIP (Anivsa, 2017). Este cuidado é necessário, pois a presença de umidade no sitio inserção durante a CIP, viola a barreira de proteção presente na pele, favorecendo a proliferação de microorganismos e a disseminação dos mesmos (Silva; Arreguy-Sena, 2014). A não implicação desses cuidados pode resultar em uma antissepsia ineficiente, podendo evoluir para infecções da corrente sanguínea e outras complicações no decorrer da TIV.

Com relação aos cuidados clínicos após a inserção de CIP em crianças hospitalizadas, no presente estudo 91,9\% das CIP foram estabilizadas imediatamente após o sucesso da CIP, sendo que os profissionais utilizaram o estabilizador denominado o IV - FIX ${ }^{\circledR}$. Este estabilizador apesar de ser estéril, não dá visibilidade ao sitio de inserção, impedindo a monitorização do mesmo. Também foi observado que os profissionais responsáveis pela CIP associavam o uso do estabilizador estéril as coberturas não estéreis, tais como a fita adesiva do tipo esparadrapo e micropore ${ }^{\circledR}(95,1 \%)$. Dados semelhantes foram encontrados em um estudo também feito em Feira de Santana (Santos et al., 2013) cujo objetivo foi analisar os critérios para a fixação de acessos venosos periféricos. Não se recomenda a utilização de fitas adesivas e suturas para estabilizar cateteres periféricos, pois podem ser facilmente contaminados com microorganismos patogênicos (Anvisa, 2017).

Diante disso, as práticas do presente estudo não estão de acordo com o que é preconizado pela literatura. A técnica deve ser corrigida, pois além de ir contra os princípios da prática de segurança do paciente, pode contribuir com aparecimento complicações locais associadas à TIV. Dessa forma, para garantir e promover a segurança no uso de dispositivos vasculares periféricos, a equipe de enfermagem precisa incorporar em seus cuidados clínicos a utilização do bundle que foi criado, para obter práticas de maior qualidade.

\section{CONSIDERAÇÕES FINAIS}

O estudo possibilitou avaliar os cuidados clínicos dos profissionais de enfermagem antes, durante e após a inserção de cateteres intravenosos periféricos em crianças hospitalizadas na unidade de clínica médica do HEC em Feira de Santana na Bahia. Revelou que os profissionais deixaram de adotar medidas básicas para a prevenção de 
infecções relacionadas à CIP. Na seleção do sítio de inserção observou-se que os profissionais possuem boas práticas, porém em relação aos cuidados durante a inserção do cateter, no que se refere ao preparo do sitio de inserção, a técnica asséptica da pele não está de acordo com o que é recomendado, não esperam o tempo de secagem da solução e ainda tocam no sítio após a antissepsia da pele. Entretanto utilizam soluções antissépticas corretas, o álcool à $70 \%$ e a clorexidina alcoólica. Após a CIP, os profissionais estabilizam o $h u b$ do cateter de imediato e com a tecnologia adequada, porém associam com fitas adesivas do tipo esparadrapo e Micropore $^{\circledR}$, com a ideia de que reforça a fixação, porém compromete a estabilização.

Perante os fatos, fica claro que estes profissionais não possuem padronização dos cuidados clínicos antes, durante e após a realização da CIP, necessitando da utilização de protocolos baseados em evidencia, a exemplo do blunde que foi construído, que poderá reduzir a irregularidade nos cuidados clínicos prestados pelos profissionais de enfermagem. Este bundle irá contribuir de forma positiva para o hospital, pois irá prevenir complicações relacionadas ao momento da CIP, otimizar o trabalho e reduzir os gastos desnecessários com novas CIP ou tratamento das complicações decorrentes aos cuidados clínicos ineficaz.

\section{REFERÊNCIAS}

AGENCIA NACIONAL DE VIGILANCIA SANITARIA (ANVISA). 2017. Segurança do Paciente e Qualidade em Serviços de Saúde. Medidas de Prevenção de Infecção Relacionada à Assistência à Saúde. Distrito Federal - DF.

ALMEIDA, T.J.C. et al. 2016. Acessos venosos periféricos em crianças hospitalizadas: um estudo fotográfico. Revista de enfermagem UFPE on line-ISSN: 1981-8963, v. 10, n. 2, p. 701-707.

AVELAR, A.F.M.; PETERLINI, M.A.S.; PEDREIRA, M.L.G. 2013. Assertividade e tempo de permanência de cateteres intravenosos periféricos com inserção guiada por ultrassonografia em crianças e adolescentes. Revista da escola de enfermagem. USP, v. 47, n. 3, p. 539-546.

BATISTA, O.M.A. et al. 2014.Fatores de risco para as complicações locais da terapia intravenosa periférica. Revista enfermagem UFPI, v. 3, n. 3, p. 88-93.

INFUSION NURSES SOCIETY BRASIL (INS - BRASIL). 2013. Diretrizes práticas para a terapia infusional. São Paulo.

INFUSION NURSES SOCIETY. 2016. Infusion Therapy Standards of Practice. Supplement to Journal of Infusion nursing, v. 39, n. 1S.

INSTITUTE FOR HEALTHCARE IMPROVEMENT (IHI). 2012. Getting Started Kit: Prevent Central Line Infections How to Guide. Cambridge: Institute for Healthcare Improvement.

LOURENÇO, S.A.; DA SILVA OHARA, C.V. 2010. Conhecimento dos enfermeiros sobre a técnica de inserção do cateter central de inserção periférica em recém-nascidos.

Revista Latino-Americana de Enfermagem, v. 18, n. 2, p. 189-195. 
MARTINS, K.A. et al. 2008. Adesão às medidas de prevenção e controle de infecção de acesso vascular periférico pelos profissionais da equipe de enfermagem. Ciência,

Cuidado e Saúde, v. 7, n. 4, p. 485-492.

MODES, P.S.S.A. et al. 2011. Cuidados de enfermagem nas complicações da punção venosa periférica em recém- nascidos. Revista Rene, Fortaleza, Abr.-Jun.

OLIVEIRA, M.I.V.; BEZERRA, M.G.A.; PEREIRA, V.R. 2016. Cateterização venosa: assistência de enfermagem-UTI pediátrica. Northeast Network Nursing Journal, v. 9, n. 2.

SANTOS, L.M.D. et al. 2013. Critérios para a fixação de acessos venosos periféricos em recém-nascidos prematuros. Revista de pesquisa cuidado é fundamental (Online), v. 5, n. 1, p. 3238-3250.

SILVA, R.N.A.; ARREGUY-SENA, C. 2014. Trauma vascular periférico em crianças: fatores relacionados pelo método de regressão logística. Revista Eletrônica de Enfermagem, v. 16, n. 1, p. 117-24. 